\title{
Development of Protein Biomarkers for the Authentication of Organic Rice
}

\author{
Ju-Young Lee ' Jinkyu Lim* \\ Received: 17 August 2015 / Accepted: 11 September 2015 / Published Online: 31 December 2015 \\ (C) The Korean Society for Applied Biological Chemistry 2015
}

\begin{abstract}
The rice protein profiles of Oryza sativa L (Koshihikari) grown under organic and conventional cultivation regimes were compared on 2-D gels to develop diagnostic marker proteins for organic rice. The selected proteins, differentially expressed between organic and conventional rice, were compared with the differentially expressed proteins of another organic and conventional rice pairing, produced at a different location. In the first comparison among conventional, no-chemical, and organic rice grown in the same region, Korea, 13 proteins exhibiting differential expression in organic and conventionally grown plants were selected. Eight of the 13 proteins were down-regulated, and the 5 remaining proteins were up-regulated from conventional to organic rice. The second comparison pairing from Kyungju, revealed 12 differentially expressed proteins, with 8 down-regulated and 4 up-regulated proteins. Ten of the differentially expressed proteins that overlapped between the two comparison sets could not be clustered into any functional group using a functional annotation clustering tool. Further comparisons using another set of conventional and organic rice, belonging to a different variety of Oryza sativa $\mathrm{L}$ and produced in Sanchung, revealed 8 differentially expressed proteins, 5 of which were down-regulated and 3 of which were upregulated in the organic rice. Overall, 3 differentially expressed proteins were commonly found in all three organic rice crops. These 3 proteins, along with other overlapping differentially expressed proteins, can provide a good starting point for the development of signature proteins that can be used for the authentication of organic rice with a follow-up studies with more comparison sets.
\end{abstract}

J.-Y. Lee · J. Lim

Department of Food Biomaterials, College of Agriculture and Life Sciences, Kyungpook National University, Daegu 702-701, Republic of Korea

*Corresponding author (J. Lim: jkylim@knu.ac.kr)

This is an Open Access article distributed under the terms of the Creative Commons Attribution Non-Commercial License (http://creativecommons. org/licenses/by-nc/3.0/) which permits unrestricted non-commercial use, distribution, and reproduction in any medium, provided the original work is properly cited.
Keywords 2D gel $\cdot$ authentication $\cdot$ conventional $\cdot$ differential expression · endosperm · organic · Oryza sativa $\mathrm{L} \cdot$ rice soluble protein

\section{Introduction}

According to the United States Department of Agriculture (USDA) definition, organic food is made with more than $95 \%$ certified organic ingredients, and using organic methods (USDA, 2015). The organic food market in Korea grew at an average rate of 50\% from 2006 to 2011, and is expected to expand to $\$ 6$ billion by 2020 (Organic_Trade_Association, 2015). Consumers are becoming more and more interested in organic agricultural products, mainly because of food safety and nutritional quality concerns; however, there are no data that support the public opinion that organic food is of a higher nutritional quality than conventionally produced food (Bourn and Prescott, 2002). In addition, consumers of organic produce contribute to the preservation of natural resources and biodiversity, and to the support of animal health and welfare, as they favor these values, regardless of their association with higher food prices. Thus, farmers attempt to enter the organic farming industry, attracted by the prospect of selling their products at premium prices and in increasingly expansive markets, in spite of the industry's strict standards and regulations and the lower yields seen in the production of organic agriculture products. However, the genuine efforts made by organic rice producers to preserve the environment and provide healthy food to consumers can easily be discouraged by the adulteration of organic rice with conventional rice, and the use of falsified labels. Conventional produce falsely labeled as organic has also discouraged consumers from purchasing organically grown products. For this reason, differentiation and certification methods developed for the authentication of organic products can promote and improve organic farming.

There have been many attempts to develop methods for identifying organic produce: developing "diagnostic" genes from 
high- and low-input agricultural regimes for the authentication of organic wheat via microarray analysis (Lu et al., 2005), identifying "diagnostic" proteins using 2D gels and the mass analysis of conventional and organic wheat (Zorb et al., 2009), and potato (Lehesranta et al., 2007) have shown that the authentication of organic produce could possibly be accomplished by establishing the specific protein signatures of produce grown under organic or conventional regimes. In addition, metabolomic fingerprinting has demonstrated that analytical tools can potentially be used for organic food authentication (Novotna et al., 2012; Gao et al., 2013; Wang et al., 2013b). However, the substantial fluctuations in the metabolome profiles of the crops due to differences in cultivation conditions can make metabolomic comparisons carried out in the interest of authenticating organic produce very complicated, and to some extent, meaningless. Meanwhile, although the protein content of a plant also changes in response to environmental conditions, such as, dryness (Pandey et al., 2010), salinity (Liu et al., 2012), temperature (Han et al., 2009; Gammulla et al., 2011), pathogenic stress (Wang et al., 2013a), changes in developmental phase (Yang et al., 2006; Hamamoto et al., 2012), the proteome profiles of the plant tissues, especially rice endosperm, are relatively comparable between the input groups (Lee et al., 2012).

Of the major global foods produced by monocotyledonous plants, rice is not only a staple crop, but also a model monocot for molecular biological studies. The proteomic changes that occur in rice grown under different culture conditions and at different growth stages have been studied in detail. However, very limited data have been published on the proteome profile of the starchy endosperm of white rice, which is the primary edible part of the plant and the form of rice most readily available to consumers.

Thus, in this study, the proteomic analysis of rice endosperm using 2 dimensional gel electrophoresis and matrix assisted laser desorption ionization mass spectrometry was carried out to elucidate the effects of organic and conventional cropping methods, and to further develop organic farming-specific biomarkers for rice. Rice samples from plants grown under two different organic and conventional farming regimes at two different locations were used to compare the protein profiles among treatments, using 2-D gels. To confirm the validity of the selected biomarkers, we compared conventional and organic rice of a different variety of Oryza sativa $\mathrm{L}$, grown in another area.

\section{Materials and Methods}

Sample collection and protein preparation. Rice sample collection: The rice samples used in this study were collected from three different organic rice farms located in Kyungju, Yeoju, and Sanchung, which are located in the south-eastern, middle, and south-western regions of Korea. Three commercial Ministry of Agriculture, Food and Rural Affairs (MAFRA), Korea certified organic and conventional rice samples (cultivar Koshihikari) were purchased directly from the producers.
Extraction of rice proteins for 2-D gel analysis: To extract proteins from the rice, the white rice was ground in a blender, and $10 \mathrm{~g}$ of the powder was soaked in $100 \mathrm{~mL}$ of distilled water for 2 $\mathrm{h}$ at room temperature. The soluble rice protein in the supernatant fraction was recovered via centrifugation at $10000 \times g$ for $20 \mathrm{~min}$ at $4^{\circ} \mathrm{C}$. The protein concentration was measured using the Bradford reagent (BioRad, USA), with a standard curve based on BSA. For the 2-D gel analysis, proteins were precipitated using $10 \%$ trichloroacetic acid (TCA), for $1 \mathrm{~h}$ at $4^{\circ} \mathrm{C}$. Precipitated proteins were centrifuged for $10 \mathrm{~min}$ at $15000 \times \mathrm{g}$. The protein pellet was resuspended in $100 \%$ ethanol, vortexed for $5 \mathrm{~min}$ and centrifuged for $5 \mathrm{~min}$ at $10000 \times \mathrm{g}$. This washing was repeated for three times. Likewise, the washed pellet was resuspended in $70 \%$ ethanol and centrifuged for a final wash. The protein collected from the final wash was freeze-dried for $1 \mathrm{~h}$. The washed and dried pellet was dissolved in a rehydration solution containing $7 \mathrm{M}$ urea, $2 \mathrm{M}$ thiourea, 4\% 3-[(3-cholamidopropyl)dimethylammonio]-1-propanesulfonate, $1 \mathrm{M}$ dithiothreitol, and $0.5 \%$ Ampholyte $\mathrm{pH} 3-10$ (BioRad) for $2 \mathrm{~h}$ at $30^{\circ} \mathrm{C}$, ultra-sonicated for $10 \mathrm{~min}$ in an ice bath, and centrifuged for $10 \mathrm{~min}$ at $15000 \times \mathrm{g}$ for clearing. The soluble proteins were re-precipitated with $10 \%$ TCA, washed with ethanol, dried, and re-dissolved in the rehydration solution to remove bound ions from the proteins. As with other plant proteins (Nawrocki et al., 2011), the double precipitation and extensive wash steps were essential to obtain high-resolution 2-D gels. The gel analysis for each sample was repeated at least two times to ensure the reproducibility of the comparisons.

2-D gel and image analysis. Two dimensional gel analyses were performed according to the method described by Lee et al. (2012). Briefly, $450 \mu \mathrm{g}$ of proteins dissolved in rehydration solution are added to a $17 \mathrm{~cm}-$ Immobilized pH Gradient strip (pH 4-7, BioRad), and focused using a focusing program of $40000 \mathrm{Vh}$ at a maximum voltage of $10000 \mathrm{~V}$. The second-dimension separation of the proteins was performed on sodium dodecyl sulfate polyacrylamide gel electrophoresis using 12\% polyacrylamide gels. Proteins were stained for $2 \mathrm{~h}$ in Coomassie Brilliant Blue R-250, in 45\% methanol and $7.5 \%$ acetic acid in deionized water. The stained gels were then de-stained in $40 \%$ methanol and $10 \%$ acetic acid in deionized water. Digitalized images of the proteins were obtained using a scanning device (Epson Perfection V-700 scanner, Japan). Gel images were cropped and analyzed using the PDQuest software program (BioRad). The intensities and positions of spots on the gels were normalized using several constitutively expressed protein spots. Due to variations in sample quantities loaded and the quality of the IPG strips, spot intensities in different gels needed to be normalized by equalizing the intensities of \#73 proteins in addition to the calibration of the spot intensities by adjusting the gaining of the background using PDQuest software. After normalization, Gaussian images were obtained from the comparison gels. A Gaussian gel image was used to find differentially expressed protein spot on comparison gels.

Identification of proteins via peptide mass fingerprinting. Using automatic spot detection and matching and semiautomatic spot editing, differentially expressed proteins exhibiting greater 
than two fold differences in expression between conventionally and organically grown plants were selected and manually excised from gels using scalpels. The proteins in the gel pieces were destained and trypsinized as described by Lee et al. (2012). The resulting peptides were extracted, dried, and dissolved in a mixture of deionized water:acetonitrile:trifluoroacetic acid (TFA) (93:5:2). A 1:1 mixture of peptides and a matrix solution (10\% $\alpha-$ cyano-4-hydroxycinnamic acid, 50\% methanol, $0.1 \%$ TFA) spiked with internal standards (bradykinin, $\mathrm{m} / \mathrm{z}=904.4681$ and angiotensin $1, \mathrm{~m} / \mathrm{z}=1296.6853$ ) was spotted and crystalized on a MALDI plate. The mass values of the peptides at each protein spot were measured using a MALDI-Time-of-Flight (TOF) MS (Voyager-DE STR, PerSeptive Biosystems, USA) with a range of 850-3000 Da. Proteins were identified by searching their mass values against NCBI and Swiss-Prot public databases using the search engines MS-Fit (http://prospector.ucsf.edu/prospector/ mshome.htm) and Mascot (Matrix Science, UK) for confirmations, respectively. Information about the proteins' functions was obtained by searching databases with the accession numbers of the proteins using UniProt (http://www.uniprot.org/uniprot/). The functional groupings of the selected proteins were analyzed using the DAVID Annotation Clustering Tool ver. 6.7 (Huang and Lempicki, 2009a; 2009b) (http://david.abcc.ncifcrf.gov/).

\section{Results and Discussion}

Agricultural regime and rice protein profile changes. Assayed by micro-Kjeldahl analysis (Miller and Houghton, 1945), the total protein content of the samples from Yeoju, Kyungju, and Sanchung were determined to be $6.21,6.01$, and $6.38 \%$ for organic rice, and $6.34,6.22$, and $6.75 \%$ for conventional rice, respectively. The water soluble protein content of the samples was determined via the Bradford method (Bradford, 1976), and found to make up approximately $40 \%$ of the total protein content. These results suggest that agricultural regimes, including nitrogen input, soil, and temperature changes affect the expression levels of proteins in the endosperm of rice, lowering the protein content of organic rice compared to that of conventional rice (Zorb et al., 2009). However, although the total protein content of conventional rice was higher than that of organic rice, the overall protein profiles as visualized on the 2-D gels were not significantly different (Fig. $1,3)$. Thus, the gel image sets used for the selection of organic rice-specific protein markers were comparable. This phenomenon was also observed in rice grown under two different conventional regimes which included different temperature fluctuations and $\mathrm{N}$ inputs during the cultivation period (Lee et al., 2012).

The representative protein profiles of rice grown under different regimes (conventional, no-chemical, and organic farming) from Yeoju, revealed approximately 450 proteins on the 2D gels (Fig. 1). Functional analysis via peptide mass-fingerprinting using MALDI-TOF MS identified 111 proteins out of the 450 protein spots visible on the gels (Fig. 2 and Supplementary Table 1). The differentially expressed proteins were labeled using an alphabetical initial denoting the region in which the samples were grown was assigned, along with a number to allow differentiation among spots in samples from each region (Fig. 2). For example, protein spot number 19 from Yeoju rice was designated Y19, while Kyungju rice spot number 19 was labeled K19, etc. Prior to the comparison of the individual proteins with those on the other 2-D gels, the loading amount of the proteins on each gel should be normalized. Thus, in addition to adjusting the spot intensities by calibrating gaining functions, the spot intensities of the proteins were normalized by adjusting the intensity of spot \#73, which is consistently expressed in similar amount in all samples, using the image-analysis software PDQuest. The functions of the differentially expressed proteins were annotated by searching databases using the search engines, Uniprot (www.uniprot.org) and DAVID (see Materials and Methods) (Supplementary Table 1). Among a set of proteins whose expression levels differed between conventional

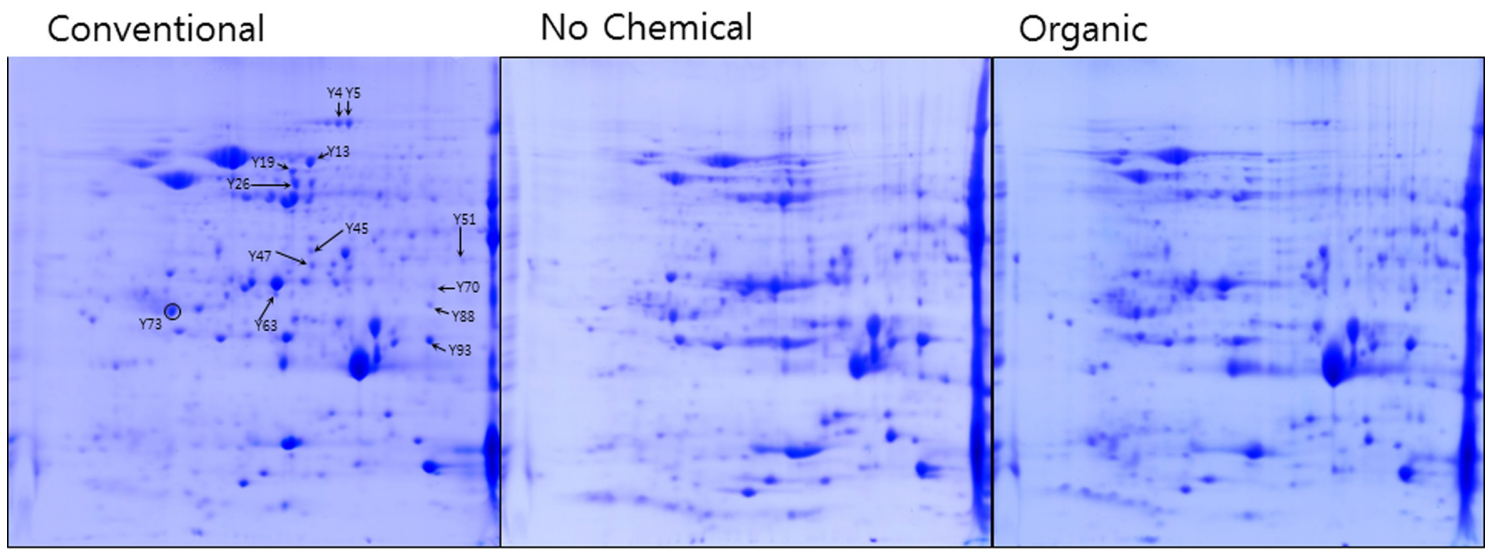

Fig. 1 Comparison of rice proteins from conventional, no chemical, and organic rice on 2-D gels. Proteins extracted from rice from three different cultivation regimes (conventional, no chemical, and organic) in the Yeoju area were separated on 2-D gels. The spot intensities on different gels were normalized via the equalization of the intensity of protein spot \#73 on each gel, using the image- analysis software program PDQuest. Differentially expressed proteins, either down-regulated or up-regulated in organic rice, were selected using painstaking image analysis comparison procedures and marked with arrows and numbers preceded with a Y to designate the samples' cultivation area. 
Table 1 List of differentially expressed proteins selected via comparisons of organic and conventional rice

\begin{tabular}{|c|c|c|c|c|c|}
\hline Spot \# & MOWSE Score & $\% \mathrm{Cov}$ & $\begin{array}{l}\text { Accession } \\
\text { Number }\end{array}$ & Protein Name & Protein Function \\
\hline 4 & 66901 & 12.8 & Q7EYV7 & Poly [ADP-ribose] polymerase & DNA repair \\
\hline 5 & 5225 & 9.7 & Q7EYV7 & Poly [ADP-ribose] polymerase & DNA repair \\
\hline 8 & 11927 & 7.1 & Q7Y001 & PINHEAD & translational regulation miRNA binding \\
\hline 13 & 124130 & 12.2 & $\mathrm{~A} 2 \mathrm{Z} 1 \mathrm{C} 8$ & Cellulose synthase A catalytic subunit & cell wall biosynthesis \\
\hline 19 & 81254 & 13.4 & Q2QZ80 & Laccase-21 & lignin degradation \\
\hline 22 & $4.15 \mathrm{E}+13$ & 46.5 & Q53LQ0 & Protein disulfide isomerase-like 1-1 & protein folding \\
\hline 26 & $2.39 \mathrm{E}+11$ & 26.8 & Q65XK0 & Ketol-acid reductoisomerase & amino acid metabolism \\
\hline 31 & $2.86 \mathrm{E}+15$ & 52 & Q42971 & Enolase & glycolysis \\
\hline 45 & $2.22 \mathrm{E}+07$ & 35.2 & Q7XDC8 & Malate dehydrogenase, cytoplasmic & metabolism \\
\hline 47 & 38289 & 21.3 & P41095 & $60 \mathrm{~S}$ acidic ribosomal protein $\mathrm{P} 0$ & translation \\
\hline 51 & 26521 & 17.7 & Q5JNA1 & B3 domain-containing protein & DNA repair \\
\hline 58 & $3.90 \mathrm{E}+08$ & 41.2 & Q948T6 & Lactoylglutathione lyase & conversion of hemimercaptal, S-lactoylglutathione \\
\hline 59 & 4488 & 21 & Q948T6 & Lactoylglutathione lyase & conversion of hemimercaptal, S-lactoylglutathione \\
\hline 63 & 23082 & 8.4 & Q0JKV1 & Potassium channel AKT1 & ion transport \\
\hline 70 & $1.66 \mathrm{E}+08$ & 49.7 & Q53NL5 & Xylanase inhibitor protein & defense against secreted fungal pathogen xylanases \\
\hline 80 & 3406 & 11.7 & Q10MH8 & \multicolumn{2}{|c|}{ DEAD-box ATP-dependent RNA helicase hydrolase } \\
\hline 88 & $2.32 \mathrm{E}+05$ & 43.6 & Q69V23 & Cellulose synthase A catalytic subunit & cell wall biosynthesis \\
\hline 92 & 393150 & 25.8 & P29835 & $19 \mathrm{kDa}$ globulin & storage \\
\hline 93 & $3.10 \mathrm{E}+06$ & 43.6 & P0C5C9 & 1-Cys peroxiredoxin $\mathrm{A}$ & antioxidant protein \\
\hline 94 & 19945 & 25.8 & P29835 & $19 \mathrm{kDa}$ globulin & storage \\
\hline 102 & 5747 & 24.7 & Q01882 & Seed allergenic protein RAG2 & allergen \\
\hline 110 & 42908 & 25 & Q0JMY1 & Poly synthetase 2-B & DNA repair \\
\hline
\end{tabular}

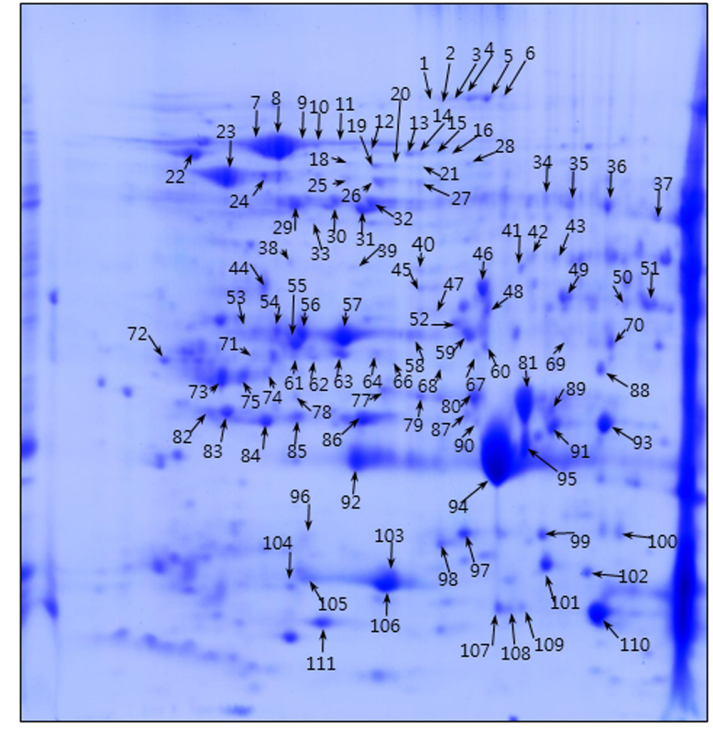

Fig. 2 Representative protein profile of rice on 2-D gels. Proteins from Oryza sativa L. were separated using $17 \mathrm{~cm}$ SDS-PAGE. One hundred and twelve of about 450 protein spots could be identified via peptide mass fingerprinting, and are indicated by arrows and numbers. The functional annotation of the proteins is shown in Supplementary Table 1.

and organic samples from Yeoju by a factor of $>2,8$ proteins underwent a gradual decrease in expression from that observed in conventionally grown rice, to that of no-chemical rice, and finally to that of organic rice. Conversely, the differential expression of 5 proteins increased from conventionally grown rice, to organic rice (Fig. 1, 4A). The group of proteins whose expression levels decreased from conventional to organic rice is comprised of Poly [ADP-ribose] polymerase 1, Protein disulfide isomerase-like 1-1, cellulose synthase A subunit 9, laccase 21, ketol-acid reductoisomerase, malate dehydrogenase, $60 \mathrm{~S}$ acidic ribosomal protein, and seed allergen protein. The group of proteins whose expression levels increased is made up of a B3 domain-containing DNA binding protein, potassium channel AKT1, a xylanase inhibitor, probable cellulose synthase A subunit 3, and 1-cys peroxiredoxin A (Table 1).

Differential expression of proteins from conventional vs. organic rice, grown under different agricultural regimes and in different area. As agricultural regimes clearly affect protein expression (Lu et al., 2005; Lee et al., 2012), we investigated whether the differentially expressed proteins found in the endosperm of rice from Yeoju are also differentially expressed among rice cultivated under other regimes, which subject the plants to different soil conditions, weather conditions, nutritional inputs, and many other factors. The comparison of conventional and organic rice samples from the Kyungju area, which is located $250 \mathrm{~km}$ south-east of Yeoju, was carried out using 2-D gels, to investigate the differential expression of proteins among growth regimes and growing locations. After conducting an image analysis along with manual validation and matching, 8 downregulated proteins and 4 up-regulated proteins, whose expression ratios fulfill the criteria of exhibiting a more than 2 fold difference between conventional and organic rice, were selected. The functions 


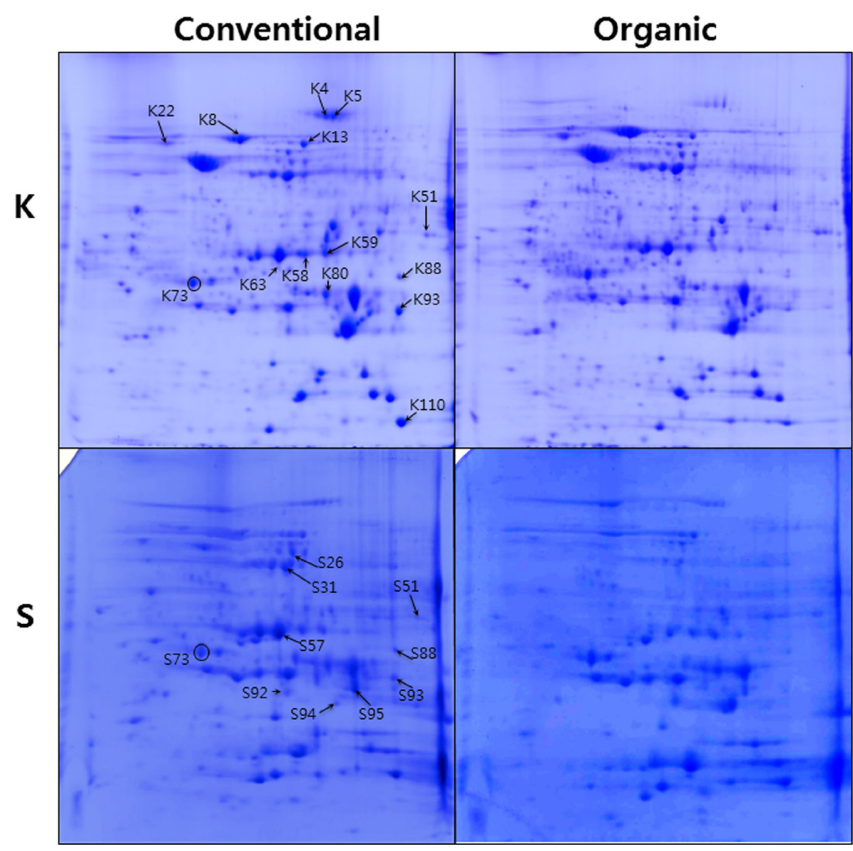

Fig. 3 Comparison of rice proteins from conventional and organic rice grown in different areas. Proteins from one conventional and organic rice comparison set, Oryza sativa L., from Kyungju (K) are compared here. The differentially expressed proteins were selected and marked with arrows and numbers, and a letter $\mathrm{K}$ to indicate the samples' area of cultivation. The other comparison set used a different variety of Oryza sativa subsp. japonica grown in Sanchung (S), and displays differentially expressed proteins designated with numbers and a letter $\mathrm{S}$ to indicate the area of origin.

of the 8 down-regulated proteins are as follows: poly [ADPribose] polymerase, cellulose synthase A catalytic subunit, lactoylglutathione lyase, DEAD-box ATP-dependent RNA helicase, probable cellulose synthase A catalytic subunit, 1-Cys peroxiredoxin A, and poly [ADP-ribose] polymerase 2-B. The functions of the 4 up-regulated proteins are as follows: protein argonaute, protein disulfide isomerase-like 1-1, B3 domain-containing protein, and potassium channel AKT1. There are 7 overlapping differentially expressed proteins between the Yeoju and Kyungju rice comparison sets. Two of the overlapping proteins down-regulated in organic rice are \#4, a poly [ADP-ribose] polymerase, which is responsible for base excision repair, and \#13, a cellulose synthase A catalytic subunit, which is involved in cell wall synthesis or non-cellulosic polysaccharide synthesis. Two overlapping differentially expressed proteins that are up-regulated in organic rice are $\# 51$, a B3 domain-containing protein, and \#63, a potassium channel AKT1. These proteins are involved in transcription regulation and ion transport, respectively. The other two inversely overlapping proteins, \#88, cellulose synthethase A, and \#93, 1-cys peroxiredoxin A, which are active in non-cellulosic polysaccharide synthesis and growth regulation during anti-oxidative stress, respectively, are down-regulated in organic rice grown in Kyungju. However, in the Yeoju comparison set, these proteins are up-regulated in organic rice. Conversely, Y22 and K23, which are protein
(A)

(B)

(C)
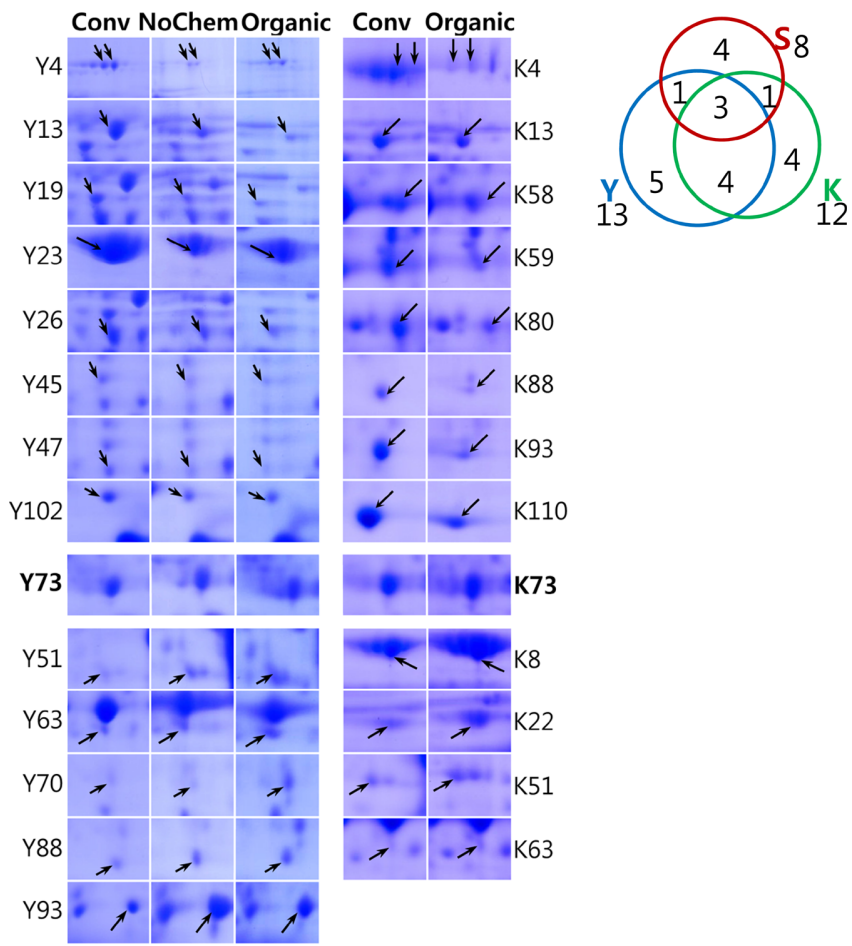

Fig. 4 Panels of differentially expressed proteins detected in organic rice. (A), Proteins down-regulated (arrows downward) and up-regulated (arrows upward) in organic rice from Yeoju (Y) are compared as panels of protein spots from conventional (Conv), no chemical (NoChem), and organic rice. Normalization of the spot intensities of the protein on 2-D gels was conducted by equalizing the spot intensities of the \#73 proteins. (B), differentially expressed proteins from the Kyungju (K) comparison set. (C), a Venn diagram of overlaps between differentially expressed proteins in the different comparison sets. Overlaps of 13 differentially expressed proteins in organic rice from Yeoju (Y), 12 from Kyungju (K), and 8 from Sanchung (S) are shown.

disulfide isomerase-like 1-1, are down-regulated in organic rice grown in Yeoju, but up-regulated in Kyungju organic rice. As mature grain is the final product of cultivation, which is influenced by multiple environmental factors, cis- and/or transacting elements can reciprocally change the expression level of specific genes (Guo et al., 2004). Thus, it is possible that the expression levels of Y88 and Y93 in organic rice are downregulated, while Y23 is up-regulated compared expression levels observed in conventional rice. However, in organic Kyungju rice, the expression patterns of K88, K93, and K22 is the opposite of those observed in rice from Yeoju (Fig. 4A, B). The seven overlaps between 12 and 13 differentially expressed proteins found in rice grown under different cultivation regimes strongly support our attempts to identify proteins suitable for use in identifying organically produced rice. In addition, we investigated whether the selected differentially expressed proteins from Yeoju and Kyungju rice were also differentially expressed in a different variety of Oryza sativa L., produced under a third cultivation regime. 
Differentially expressed proteins from a different cultivar of Oryza sativa L. cultivated under different regimes. To evaluate the overlapping proteins from the two different cultivation regimes, at Yeoju and Kyungju, for the suitability of their use in the authentication of organic rice, proteins from a different variety of Oryza sativa L., cultivated under organic and conventional regimes in Sanchung, $(200 \mathrm{~km}$ south from Yeoju and $120 \mathrm{~km}$ west from Kyungju), were compared on 2-D gels. Eight proteins were found to exhibit more than 2-fold difference in expression between the conventional and organic cultivation conditions. These proteins were then selected and identified in Sanchung rice, and compared to the differentially expressed proteins from the Yeoju and Kyungju comparison sets. Four of the 8 differentially expressed proteins from Sanchung organic rice overlapped with the differentially expressed proteins found in Kyungju rice. In organic rice, S57, S88, and S93 are down-regulated, while S51 is up-regulated. Meanwhile, 4 overlapping differentially expressed proteins from Yeoju and Sanchung rice are down-regulated at \#26 and up-regulated at \#51, and inversely regulated at \#88 and \#93 (i.e., up-regulated in Yeoju but down-regulated in Sanchung organic rice).

Overall, there are 3 commonly overlapping proteins found in all three comparison sets: $\# 51, \# 88$, and \#93, a B3 domain-containing protein, cellulose synthase A catalytic subunit 3, and 1-cys peroxiredoxin A, respectively. Plus, S26, a Ketol-acid reductoisomerase, overlaps with Y26 while S57 (lactoylglutathione lyase) overlaps with K58. According to the functional analysis, these proteins seem to be differentially expressed during the early stages of endosperm formation, because only actively dividing cells require cell wall synthesis, DNA repair, amino acid synthesis, and anti-oxidation. This suggests that although differential protein expression is influenced by multi-factorial inputs during the endosperm formation of rice, some of the proteins are commonly involved in responses the various challenges of different cultivation regimes. These common responders can be used to develop diagnostic markers for organic rice.

Suitability of Proteins for Authentication of Organic Rice. Of the total 33 proteins that are differentially expressed between organic and conventional rice from three different cultivation regimes and two different varieties of Oryza sativa L., 3 proteins were found to overlap in all three comparison sets (Fig. 4C). These appearances strongly suggest that the differential expression of the common proteins is somehow influenced by the different growing conditions present under organic and conventional agricultural regimes. Thus, they are good candidates for use as diagnostic markers designed to enable the authentication of organic rice.

Of course, analyzing approximately 450 protein spots from two varieties of rice grown under three different regimes represents too small sample size, especially considering the many different varieties of rice and agricultural regimes. This feasibility study uses a limited sample size and a small number of cultivation conditions, and thus the validity of our findings should be strengthened with follow up studies lasting several years and investigating more rice varieties, to establish more robustly supported diagnostic proteins that can be used to authenticate organic rice with confidence. However, the information on the differentially expressed proteins obtained from the rice grains in this study can be used to complement previously published diagnostic protein or metabolite data used for the authentication of organic rice.

For example, the differentiation between organic rice and adulterated or conventional rice can be carried out by examining the differentially expressed specific marker protein profiles, and the relative ratios of the marker protein intensities visualized on 2$\mathrm{D}$ gels. Other attempts to authenticate organic produce using metabolite analysis via gas chromatography-tandem MS (Wang et al., 2013b) or even an electronic nose (Peng et al., 2015) can also complement each other and together confirm the suitability of the selected diagnostic markers.

Acknowledgments This research was supported by the Basic Science Research Program through the National Research Foundation of Korea (NRF), funded by the Ministry of Education (NRF-2011-0012683) and the Kyungpook National University Research Fund, 2012.

\section{References}

Bourn D and Prescott J (2002) A comparison of the nutritional value, sensory qualities, and food safety of organically and conventionally produced foods. Critl Rev Food Sci Nutri 42, 1-34.

Bradford MM (1976) A rapid and sensitive method for the quantitation of microgram quantities of protein utilizing the principle of protein-dye binding. Anal Biochem 72, 248-54.

Gammulla CG, Pascovici D, Atwell BJ, and Haynes PA (2011) Differential proteomic response of rice (Oryza sativa) leaves exposed to high- and low-temperature stress. Proteomics 11, 2839-50.

Gao B, Lu Y, Sheng Y, Chen P, and Yu LL (2013) Differentiating organic and conventional sage by chromatographic and mass spectrometry flow injection fingerprints combined with principal component analysis. $J$ Agric Food Chem 61, 2957-63.

Guo M, Rupe MA, Zinselmeier C, Habben J, Bowen BA, and Smith OS (2004) Allelic variation of gene expression in maize hybrids. Plant Cell Physiol 16, 1707-16.

Hamamoto K, Aki T, Shigyo M, Sato S, Ishida T, Yano K et al. (2012) Proteomic characterization of the greening process in rice seedlings using the MS spectral intensity-based label free method. J Proteome Res 11, 331-47.

Han F, Chen H, Li XJ, Yang MF, Liu GS, and Shen SH (2009) A comparative proteomic analysis of rice seedlings under various high-temperature stresses. Biochim Biophys Acta 1794, 1625-34.

Lee JY, Lee JW, Kim YR, Yeom YJ, and Lim J (2012) Proteomic changes in Odae polished white rice grown at different cultivation conditions. $J$ Appl Biol Chem 55, 79-83.

Lehesranta SJ, Koistinen KM, Massat N, Davies HV, Shapherd LV, McNicol JW et al. (2007) Effects of agricultural production systems and their components on protein profiles of potato tubers. Proteomics 7, 597-604.

Liu CW, Hsu YK, Cheng YH, Yen HC, Wu YP, Wang CS et al. (2012) Proteomic analysis of salt-responsive ubiquitin-related proteins in rice roots. Rapid Comm Mass Spectrom 26, 1649-60.

Lu C, Hawkesford MJ, Barraclough PB, Poulton PR, Wilson ID, Barker GL et al. (2005) Markedly different gene expression in wheat grown with organic or inorganic fertilizer. Proc Biol Sci 272, 1901-8.

Miller L and Houghton JA (1945) The micro-Kjeldahl determination of teh nitrogen content of amino acids and proteins. J Biol Chem 159, 373-83.

Nawrocki A, Thorup-Kristensen K, and Jensen ON (2011) Quantitative 
proteomics by 2DE and MALDI MS/MS uncover the effects of organic and conventional cropping methods on vegetable products. $J$ Proteomics 74, 2810-25.

Novotna H, Kmiecik O, Galazka M, Krtkova V, Hurajova A, Schulzova V et al. (2012) Metabolomic fingerprinting employing DART-TOFMS for authentication of tomatoes and peppers from organic and conventional farming. Food Addit Contam Part A 29, 1335-46.

Organic_Trade_Association (2015) U.S. organic showcased at biggest trade show in Korea, Korea.

Pandey A, Rajamani U, Verma J, Subba P, Charkraborty N, Datta A et al. (2010) Identification of extracellular matrix proteins of rice (Oryza sativa L.) involved in dehydration-responsive network: a proteomic approach. $J$ Proteome Res 9, 3443-64.

Peng Q, Tian R, Chen F, Li B, and Gao H (2015) Discrimination of producing area of Chinese Tongshan kaoliang spirit using electronic nose sensing characteristics combined with the chemometrics methods. Food Chem 178, 301-5.

USDA (2015) Organic multi-ingredient foods, United States Department of Agriculture, USA.

Wang W, Chen LN, Wu H, Zang H, Gao S, Yang Y et al. (2013a) Comparative proteomic analysis of rice seedlings in response to inoculation with Bacillus cereus. Lett Appl Microbiol 56, 208-15.

Wang Z, Chen P, Yu L, and Harrington Pde B (2013b) Authentication of organically and conventionally grown basils by gas chromatography/ mass spectrometry chemical profiles. Anal Chem 85, 2945-53.

Yang P, Liang Y, Shen S, and Kuang T (2006) Proteome analysis of rice uppermost internodes at the milky stage. Proteomics 6, 3330-8.

Zorb C, Betsche T, and Langenkamper G (2009) Search for diagnostic proteins to prove authenticity of organic wheat grains (Triticum aestivum L.). J Agric Food Chem 57, 2932-7. 